BULLETIN (New Series) OF THE

AMERICAN MATHEMATICAL SOCIETY

Volume 41, Number 3, Pages 341-350

S 0273-0979(04)01026-2

Article electronically published on April 13, 2004

\title{
RENÉ THOM'S WORK ON GEOMETRIC HOMOLOGY AND BORDISM
}

\author{
DENNIS SULLIVAN
}

By the early 1950's algebraic topology had reached great heights with Serre's thesis and the calculations in the seminar of Henri Cartan of the cohomology of spaces with one nonzero homotopy group in terms of Steenrod operations. There was also the appearance of the new characteristic classes of vector bundles, Pontryagin classes ( $Z$ coefficients) and Chern-Weil classes (coefficients in $R$ ) joining those of Stiefel Whitney $(Z \bmod 2$ coefficients).

René Thom absorbed all this structure, made vigorous use of it, and added a geometric perspective that combined to revolutionize topology, manifold theory, and algebraic geometry. For the unexpected and fertile results in bordism (closed manifolds mod boundaries of manifolds) of the 1954 paper [1], Thom received the Fields Medal at Edinburgh in 1958. Many more applications of Thom's ideas came even later.

\section{Thom's isomorphism And his work on Steenrod's Problem}

Thom's (1951) thesis about spherical fibrations and cohomology [2] showed a new cohomology isomorphism between the (compact) base of a vector bundle and the one point compactification of its total space (now called the Thom space). Thom's isomorphism was effected algebraically by multiplying an arbitrary class in the total space (which deformation retracts to the base) with a certain cohomology class on the total space with compact support (now called the Thom class). Geometrically the isomorphism in terms of homology could be seen by intersecting a relative cycle in the total space $(\bmod \infty)$ with the zero section to obtain a cycle in the base.

The latter perspective shows that the Thom class of the oriented normal bundle of an oriented submanifold in an oriented manifold determines by pullback the cohomology class Poincaré dual to the homology class of the submanifold.

Thom then showed how the Stiefel Whitney classes of the vector bundle could be obtained by applying Steenrod operations to the Thom class in the Thom space and then using the Thom isomorphism to get back to the base,

$$
\left(1+S q^{1}+S q^{2}+\ldots\right)(U)=\left(1+w_{1}+w_{2}+\ldots\right) \cdot U(U=\text { Thom class }) .
$$

In the next paper (1954) 1], Thom's celebrated results about classifying manifolds up to cobordism appeared. As remarked above, the revolution these results caused earned René Thom the 1958 Fields Medal at Edinburgh. The seed of the development in the paper was a geometric question - What do homology classes look like? In particular there was Steenrod's Problem - Is every homology class in

Received by the editors February 24, 2004.

2000 Mathematics Subject Classification. Primary 55-XX, 14-XX, 57-XX.

(C)2004 American Mathematical Society 
any finite polyhedron the image of the fundamental class of a closed manifold by a map of that manifold into the polyhedron?

It is interesting that Thom made deep use of Steenrod's cohomology operations to prove both positive and negative results about Steenrod's Problem. Besides the natural attraction of the question, Thom was interested in it because of a potential relationship to a celebrated unresolved problem in algebraic geometry - Is it possible by blowing up to resolve the singularities of an arbitrary algebraic variety? (See $\S 3$.

The big step in Thom's approach to Steenrod's Problem beyond the vigorous use of the current algebraic topology was a geometric picture of duality - this time Alexander-Lefschetz-Poincaré duality. This picture is now referred to as the Pontryagin-Thom construction because of Pontryagin's use of the same idea in a related special case [3]. Besides many uses related to Thom's original one, the construction was important in the discovery of exotic spheres by Milnor [14]; the manifold classification theory (Browder-Novikov-Wall) [4, 5], [6]; the early proofs of the Atiyah-Singer index theorem [7]; and very recently in the (geometric) proof of Mumford's conjecture about the stable cohomology of Riemann surface moduli space (Madsen-Weiss) 8 .

Here is the Pontryagin-Thom construction adapted to the study of Steenrod's Problem and illustrating the basic duality between geometric covariant objects and algebraic contravariant objects. For each closed manifold $V$ mapping to a polyhedron $X$ by $f$, a covariant geometric object in $X$, one can form a contravariant algebraic object on a space dual to $X$ by the following steps: 1) Approximate $f$ by an embedding of $V$ into a neighborhood $N$ of $X$ in a high dimensional Euclidean space. 2) Collapse the boundary of $N$ to a point and map by further collapse the quotient $N / \partial N$ to the normal bundle neighborhood of $V$ with its boundary collapsed to a point $\nu / \partial \nu$ (the latter can be identified with Thom space of the normal bundle $\nu$ ). 3) Induce by the Gauss map the normal bundle of $V$ from the tautologous bundle over the Grassmannian of $k$-planes $(k=$ codimension of $V)$ and consider the associated map on Thom spaces. 4) Form the composition of 2) and 3) to get a map

$$
(N / \partial N=\text { neighborhood of } X / \text { boundary }) \stackrel{D f}{\rightarrow} \text { (universal Thom space) }
$$

which is a contravariant object on $N / \partial N$ dual to the covariant object in $X, V \stackrel{f}{\rightarrow} X$.

Conversely, given a contravariant object $N / \partial N \rightarrow$ (universal Thom space), apply the geometric Thom isomorphism with $N$ as a relative cycle transversally intersecting the zero section to obtain a covariant object, a closed manifold, say $V^{\prime}$, inside $N$ which then deformation retracts to $X$. One obtains a bijection between manifolds mapping to $X$ up to cobounding in $X \times I$ and homotopy classes of maps of $N / \partial N$ into the universal Thom space.

The beauty of this equivalence is that the homotopy class of the big map of $N / \partial N$ into the universal Thom space is completely determined by the map near $V^{\prime}$, the complete preimage of the zero section, because the entire complement of the neighborhood of $V^{\prime}$ in $N$ is mapped to a contractible part of the universal Thom space, the part near infinity.

In summary, Thom has replaced via the geometric Thom isomorphism Steenrod's geometric question - Which homology classes in $X$ are represented by manifolds? 
by a dual question in algebraic topology or stable homotopy theory - Which cohomology classes of $N / \partial N$ ( $N=$ neighborhood of $X$ in a high dimensional Euclidean space) are pullbacks of the Thom class from the universal Thom space by maps of $N / \partial N$ into that Thom space?

There are immediate negative consequences of Thom's dual formulation for Steenrod's Problem using odd primary coefficients. For these coefficients the stable cohomology of the oriented Grassmannians and thus their universal Thom spaces are spaced in every 4 th dimension. So any cohomology operation with odd primary coefficients of dimension not congruent to zero mod 4 must vanish on the Thom class of the universal Thom space and so also on any class induced from it (in $N / \partial N$ say). Steenrod himself produced many such operations, like the Bockstein of reduced $p^{t h}$ powers, e.g. $\beta P^{1}$ of dimension $(2 \mathrm{p}-1)$.

The (Alexander) dual of a cohomology operation which raises dimension is a homology operation which lowers dimensions $\left(H .(X) \simeq H^{\cdot}(N, \partial N)\right)$. So we can say that homology classes which admit nonzero homology operations (odd coefficients) of odd degree must carry singularities. Roughly there are essential singularities in the cycle which represent these nonzero homology operations.

The first example is a codimension 5 nonremovable singularity corresponding to the operation $\beta P^{1}$ for the prime $3(5=2 \mathrm{p}-1)$. This first Thom example occurs in 7-dimensional homology.

Thom then went to the positive side of Steenrod's question - again using cohomology operations constructed by Steenrod - the celebrated Steenrod squares $S q^{1}$, $S q^{2}, \ldots$. Now the situation is very different. Thom had already calculated the action of those operations on the Thom class of the universal bundle and found the Stiefel Whitney classes in the 1951 paper [2]. By calculating this action in detail Thom showed the Steenrod operations acted freely (modulo their universal relations on all spaces) on the mod 2 cohomology of the nonoriented universal Thom space. Using the viewpoint of Serre and the Cartan Seminar, Thom could deduce a stable homotopy model for the universal Thom space as a product (or one point union, which is the same in the stable range) of spaces, each with one nonzero homotopy element. There was one such factor for each partition of any integer into parts not a power of two less one.

This calculation gave two corollaries: 1) Any mod 2 homology class in any space is represented by a closed manifold (possibly nonoriented) mapping into the space. 2) The stable homotopy groups of the universal nonoriented Thom space were calculated to be a direct sum of $Z / 2$ 's with one $Z / 2$ in stable dimension $n$ for each partition of $n$ into parts, none of which is a power of two less one.

In a third discussion Thom combined the prime 2 and odd primary discussions to show that for any integral class some odd multiple (bounded by the dimension of the class) can be represented by a manifold.

This splitting of Steenrod's Problem over the primes by Thom used Serre's idea of calculating modulo a class of Abelian groups. Later these ideas led to a complete localization of unstable homotopy theory at the primes and over $Q$ [9]. Also the stable Thom spaces and Thom's arguments led to the construction of the stable homotopy category whose objects are called spectra [24]. The model idea of the Thom space for unoriented bundles was successful later again and again for oriented bundles, spin bundles, and complex bundles..., but it took a lot of hard work by Adams, Milnor, Novikov, Wall and Brown-Peterson.... 


\section{Resolving SINGUlarities AND BORDISM}

Thom's spectacular results above on Steenrod's Problem still don't say what the general homology class looks like. How bad must the singularities be? What do manifolds with singularities really look like? Thom discussed general locally compact finite dimensional manifolds with singularities in the 1969 BAMS article 10]. Thom arrived at the following picture: first the expected - a locally finite partition into manifolds (stratum) of varying dimensions, partially ordered by $X<$ $Y$ iff $X$ is contained in closure $Y$ and otherwise $X$ doesn't intersect closure $Y$; then the key new insight: each stratum has a neighborhood which is a locally trivial bundle over the stratum whose fibre is the cone on a compact manifold with singularities whose partially ordered set of strata has smaller depth.

By a further stratification one can assume the cone bundles on strata are actually trivial, e.g. in a simplical complex stratified by the open cells. Now the first singularity going down has a product normal bundle whose fibre is the cone on a closed manifold $L$. If the manifold $L$ is the boundary of a manifold $W$, one can replace each fibre, cone on $L$, by a copy of $W$ and thereby smooth over this singular stratum. Proceeding inductively, any singular cycle representing homology can be resolved by replacing the normal cones of the top singularity stratum by manifolds bounding the links unless links of singularities are encountered which are not boundaries (for more on this see [11]).

This works in general to represent all cycles by manifolds with codimension at least 5 singularities. The transversal links of the top singularity may be closed 4-manifolds which don't bound, e.g. the complex projective plane $\mathbb{C} P^{2}$ (which doesn't bound because it has odd Euler characteristic).

For example, Thom's 7-dimensional counterexamples to Steenrod's problem can be represented by a 7 -manifold with one 2 -dimensional stratum of singularities, a closed 2-manifold whose neighborhood is a cone on $\mathbb{C} P^{2}$ bundle and which represents the result of the dual operation of $\beta P^{1}$ for the prime 3. A second singularity is not required until dimension 11 [1].

Thus smoothing the singularities of manifolds with singularities leads to the idea of classifying manifolds up to cobounding or bordism, $X \sim Y$ iff $X$ union $Y$ with opposite orientation is the oriented boundary of a manifold of dimension one higher. The geometric Thom isomorphism duality of $\S 1$ already contains the answer to this classification, for by taking $X$ to be a point, the (Pontryagin) Thom construction identifies bordism classes of manifolds with homotopy classes of maps of spheres $(N / \partial N$ for $X$ a point) into the universal Thom space (stable range).

The mod 2 calculations and the calculation mod Serre's class of torsion groups leads to the two famous results of the 1954 paper: a) the oriented bordism ring $\otimes Q$ is a polynomial algebra over $Q$ on one generator in every dimension $4,8,12, \ldots ; b$ ) the nonoriented bordism ring is a polynomial algebra over $Z / 2$ on one generator in every dimension except powers of two less one 2,4,5,6,8,9,10,11,12,13,14,16...

Here the product is a Cartesian product of manifolds on the covariant side and a Whitney sum of vector bundles on the Thom space side.

We have described Thom's papers in this order because as a way to classify closed manifolds, both the definition and possible calculation of bordism were unexpected. Yet both appear naturally from Thom's analysis of Steenrod's question - What do representatives of homology classes look like? (However, Jean-Pierre Serre has pointed out that Thom also learned the definition of cobordism from the 
Pontryagin's school's attempt around 1940 and later to use it to calculate homotopy groups of spheres.)

\section{Singularities in Algebraic VARIETIES}

As mentioned above Thom was interested in singularities of algebraic varieties. Two questions Thom considered were:

a) Can singularities be resolved by blow up?

b) Can singularities in a fixed ambient space be deformed away, say locally?

Now question a) in characteristic zero was resolved affirmatively in the tour de force thesis of Hironaka (1963) [12] but is still open in positive characteristic. Ten years before Hironaka's solution Thom considered question a) in the light of Steenrod's question because algebraic varieties over $\mathbb{C}$ have fundamental homology classes and resolution of singularities implies these homology classes are represented by manifolds. Perhaps Thom's approach can shed light in the positive characteristic case of question a) (using étale homotopy theory to pass into algebraic topology) even though Hironaka's work shows Thom's obstructions are not realized for complex algebraic varieties.

Regarding question b) both René Thom and Andre Weil [27] considered the following variety in $\mathbb{C} P^{5}$, the rank one $2 \times 3$ matrices up to scaling, a 3 -dimensional variety $V$ defined by three equations (three minor determinants are zero). The complex cone on $V$ in $\mathbb{C}^{6}$ has an isolated singularity whose intersection with the unit sphere $S^{11}$ defines an element in the homotopy group of the universal Thom space for $\mathbb{C}^{2}$-bundles, $\pi_{11}\left(M U_{2}\right)$. A characteristic class calculation (see Hartshorne's book) shows this element is nonzero mod 2 and over $Q$. Thus the linking submanifold in $S^{11}$ of the singularity does not bound a stably almost complex submanifold in the twelve ball. This means the cone singularity cannot be deformed away. It also means the 3-fold in $\mathbb{C} P^{5}$ is not a hyperplane section. Thus it cannot be defined by two equations.

\section{EMBEDDED AND IMMERSED SUBMANIFOLDS REPRESENTING HOMOLOGY}

It was known classically to Poincaré that codimension one integral homology classes in a manifold are represented by embedded submanifolds. In codimension two this is also true and known to Thom's generation. In higher codimensions there are obstructions which can be described by Thom's duality picture - a homology class in $M$ is represented by a submanifold of $M$ of codimension $k$ iff the Poincaré dual cohomology class in $M$ is induced from the Thom class by a map of $M$ to the Thom space of the universal $k$-bundle.

The free structure of the rational cohomology (all the powers of the universal Thom class are nonzero for $k$ even) implies all of Thom's realization obstructions are finite. So Thom gets the result that any ray in the rational homology of any dimension in any manifold is represented by an embedded submanifold. One can add the information that the normal bundle of the submanifold may be taken to be trivial in odd codimension always and in even codimension iff the intersection of the homology class with itself is zero.

Using Thom's method and the thesis of Robert Wells [13], one can also always represent a ray in even codimension by an immersed submanifold with trivial normal bundle. 


\section{Thom's signature invariant of Bordism, EXotiC SPHERES, AND THE FORMUlaE OF HiRZEBRUCH AND AtiyAH-Singer}

A beautiful and simple argument (Thom [2]) combining Poincaré-Lefshetz duality for a $4 k+1$ manifold $W$ with boundary and the exact sequence of the pair $(W, M)$ where $M=\partial W$

$$
\rightarrow H_{2 k+1}(W, M) \stackrel{i}{\rightarrow} H_{2 k} M \stackrel{j}{\rightarrow} H_{2 k} W \rightarrow
$$

shows that the signature of the middle dimensional intersection form of $M$ is zero. (Proof: $i$ and $j$ are adjoint maps under Poincaré duality so that (image $i)=($ ker $j$ ) has $1 / 2$ dimension $H_{2 k} M$. But two $2 k$ cycles in $M$ which bound in $W$ clearly have zero self-intersection numbers. Thus (ker $j$ ) is a self-annihilating subspace of $1 / 2$ dimension, and this means the signature of the form is zero.) Thus the signature which is multiplicative for cartesian products defines a ring homomorphism to $Q$ from (oriented bordism) tensor $Q$ (Thom [1]).

One corollary of this is the celebrated Hirzebruch Signature Formula, which can be proven 25 by checking it on any string of manifolds like $\mathbb{C} P^{k}, k=2,4,6, \ldots$ which generate (as shown by Thom) the bordism ring $\otimes Q$. If my memory doesn't fail, I remember Fritz Hirzebruch telling that he received Thom's C.R.A.S. note in the morning post at the IAS in Princeton and had his signature formula by the same afternoon. The fact that Pontryagin's classes were integer classes and Hirzebruch's formulae for the signature (the $L$-polynomials $P_{1} / 3,\left(7 P_{2}-P_{1}^{2}\right) / 45, \ldots$ ) had nontrivial coefficients allowed Milnor to produce the invariants needed to prove his spheres carried exotic smooth structures [14.

A similar but more elaborate statement to Hirzebruch's formula is the AtiyahSinger Index Formula for the index of elliptic operators, which can be proven by showing the index is a cobordism $K$-theory invariant and then checking the formula on a $Q$ basis, e.g. the signature operator with coefficients in a vector bundle. This was perhaps the first proof.

The signature invariant of $4 k$-manifolds was introduced in the 1920's by Herman Weyl in a paper written (in Portuguese) for a Portuguese mathematics journal [26]. Weyl said the signature invariant should be an interesting invariant of manifolds. (Sources J-P. Serre and H.E. Winkelnkemper).

\section{Thom's COMbinatorial RATiOnal CHARACTERISTIC Classes, $K$-THEORY REFINEMENTS, INTERSECTION HOMOLOGY, AND ELLIPTIC HOMOLOGY}

In the middle 50's the characteristic classes of smooth manifolds were defined to be invariants of the tangent bundle. They could be defined (Chern-Weil) by connections, e.g. the Levi-Civita connection, as traces of even powers of the curvature tensor (real coefficients) or with $Z$-coefficients by pulling back Schubert cycles by a Whitney classifying map into the Grassmannian (Pontryagin).

Thom gave a totally new method [2] for defining characteristic classes $(Q$-coefficients) for combinatorial manifolds with certain singularities, and these objects don't have tangent vector bundles. This uses Thom's duality picture and the Serre calculation that rationally there is an equivalence between $k$ dimensionalcohomology of any finite polyhedron $X$ and homotopy classes of maps of a high suspension of $X$ into spheres,

$N$-fold suspension of $X \rightarrow N+k$ sphere, $N>>k$. 
Thom considered the geometry of the polyhedral transversal pullback, $V$ say, of a point in the $N+k$ sphere. Clearly, the (local structure of $V$ ) $\times R^{N+k}$ is just the (local structure of $X) \times R^{N}$. For example the local homology sheaves are the same with a shift. (Thus if $X$ is an oriented $n$-dimensional pseudomanifold, then $V$ is an oriented $n-k$ dimensional pseudomanifold and we have a picture of the map $H^{k} X \rightarrow H_{n-k} X$ which is cap product with the fundamental class of $\left.X\right)$. Varying the choices makes $V$ vary by a "cobordism" in $X \times I$ with similar local structure statements.

One immediate corollary is a purely combinatorial definition of Hirzebruch's $L$-classes for a polyhedron whose local homology sheaves are those of an oriented manifold ( $Q$ coefficients). Namely each $V^{n-k}$ is also a local homology manifold ( $Q$ coefficients). So it has a signature which is a "cobordism" invariant for these singularities (see the argument in $\S 5$ ), and Thom gets a signature homomorphism from $H^{k} X \rightarrow Q$ (when $n-k=O(\bmod (4))$. One obtains Thom's rational homology characteristic classes. Their Poincaré duals are the combinatorial Hirzebruch $L$-classes (because the normal bundle of $V$ in $X \times R^{n}$ is trivial, consistency can be checked with the smooth case from Hirzebruch's signature formula).

Thom's argument is so good one wants to squeeze it harder. For example, what properties of the local homology sheaves of $X$ (and therefore $V$ ) imply that $V$ has a signature which is a "cobordism" invariant? It turns out there are several interesting answers and directions to follow that are related to this question. (See also p. 230 of Springer Lecture Notes in Math. 197, p. 137 of [17], and p. 1067 of 30.)

I) Jeff Cheeger's work on $L^{2}$ harmonic forms in metric spaces with metric conelike singularities (e.g. polyhedron) leads to a picture involving an inductive choice of self-annihilating subspaces in homology to have correct $L^{2}$ boundary value problems for harmonic forms. Since the $L^{2}$ theory automatically satisfies Poincaré duality, one can repeat Thom's argument. This $L^{2}$ version of Thom's argument is not published completely as far as I know, but see 15].

II) John Morgan working independently in surgery theory found an integral refinement of the above subspace choices which also included the Arf-Kervaire invariant of quadratic forms over $Z / 2$. Unfortunately none of this was published, but see [16].

III) Happily, Goresky and MacPherson working at IHES (middle 70's) under Thom's direct influence, especially stratifications and singularities in mappings, considered geometric cycles bigraded by their degree of transversality to the local homology strata and published an extremely fruitful paper [17] about a new kind of homology called intersection homology. This new theory satisfied Poincaré duality for a large class of manifolds with singularities (including those of complex algebraic varieties) [17, and Thom's argument yielding (ordinary) homology characteristic classes goes through for these spaces (now the cap product goes from ordinary cohomology to intersection homology).

A very nice denouement to this direction III) provides a general class of cycles with singularities that have signatures using intersection homology, the Witt spaces and exactly represent $K O$ homology at odd primes [30.

The category of perverse sheaves arising from the Goresky-MacPherson paper is the natural setting for intersection homology based on Verdier Duality, and there 
has been a rich development in many algebraic directions (D-modules, representation theory, algebraic analysis of PDE,...) using these sheaves.

IV) The original argument of Thom for local homology manifolds can be refined integrally using another signature jewel besides its bordism invariance - namely the signature of $M^{4 k}$ is also defined if $M^{4 k}$ has boundary and it is additive for glueing along full boundary components (Novikov) 18]. Then one can define a signature $\bmod n$ for $Z / n$ manifolds (those whose $\partial$ is divided into $n$ isomorphic groups of components) which is a $Z / n$-bordism invariant (by Novikov additivity). All this works in the context of $Q$-homology manifolds [23].

One carries through Thom's argument with those $Z / n$ objects as well as the closed $Q$ objects to obtain two results for combinatorial $Q$-homology manifolds:

1) At the prime 2: the Thom (Hirzebruch) homology characteristic class over $Q$ has a canonical lift to integral (at 2) coefficients [16], 23].

2) At odd primes: the Thom (Hirzebruch) homology characteristic class over $Q$ has a canonical lift to KO-homology $\otimes Z[1 / 2]$ [19], 23].

Note the refinement at the prime 2 corresponds to the original result of Thom concerning Steenrod's problem that manifolds represent all homology after taking odd multiples, that is, all homology with coefficients in $Z$ (localized at the prime 2 by making odd primes into units). The $\mathrm{KO}$ at odd primes refinement is related to Thom's negative result about representatives of homology requiring singularities because of certain odd primary homology operations. Namely, if one allows all singularities which preserve the fact that $(4 k)$ cycles still have a signature which is a homology invariant for these singularities, then one obtains a homology theory which is not usual homology but an extraordinary one which is isomorphic to $\mathrm{KO}$ homology (localized at odd primes by inverting the prime 2) [20], [30].

V) Imposing specific singularities on cycles and homologies defines other extraordinary homology theories [11], [29], [31], including the Morava $K$-theories and elliptic homology [21], [28], [31]. These are related to the current chromatic activity in stable homotopy theory-elliptic theory and modular forms; see [22], 229, and the preface and references therein.

It would be interesting to find for Witten's elliptic genus all the geometric cycles parallel to those mentioned above for the L-genus and Thom's signature. As I recall, Witten's work on the elliptic genus involved consideration of the multiplicative properties of invariants of manifolds which are fibred, and this property for the signature was discussed in 32], a 1957 paper of Kodaira, and a 1961 paper of Atiyah.

Postscript: This text was originally written for the special issue of the Gazette de la Société Mathématique de France dedicated to René Thom. It was improved because of an enjoyable and interesting exchange of emails with Jean-Pierre Serre, who conscientiously critiqued an earlier version. I am grateful for that and other contacts with him and with René Thom during two decades at the IHES in France. One can only admire and appreciate the scholarship and creativity of the French mathematical tradition. 


\section{REFERENCES}

[1] Thom, R., "Quelques proprietes globales des varietes differentiables", Comm. Math. Helv. 28 (1954) 17-86. MR 15:890a

[2] Thom, R., "Espaces fibres en spheres et carres de Steenrod", Ann. Sci. Ecole Norm. Sup. (3) 69 (1952); and "Les classes caracteristiques de Pontryagin des varietes triangulees", Symp. Int. de Top. Alg. UNESCO (1959) p. 40. MR 14:1004c

[3] Pontryagin, L., Doklady Akad Nauk SSSR 34 (1942) pp. 35-37.

[4] Browder, W., "Surgery on Simply Connected Manifolds", Ergebnisse Series 65, Springer (1972). MR 50:11272

[5] Novikov, S.P., "Homotopy equivalent smooth manifolds", Translations of Amer. Math. Soc. 48 (1965) pp. 271-396.

[6] Wall, C.T.C., "Surgery on Compact Manifolds", Academic Press, 1970. MR 55:4217

[7] Atiyah, M.F., and Singer, I.M., "The index of elliptic operators: I", Annals of Mathematics, 2nd Ser., vol. 87, no. 3. (May 1968), pp. 484-530. MR 38:5243

[8] Madsen, I., and Weiss, M., "Proof of Mumford's conjecture" (authors' web page).

[9] Sullivan, D., "Genetics of homotopy theory and the Adams conjecture", Annals of Math. 100, no. 1 (1974) 1-79. MR 56:1305

[10] Thom, R., "Ensembles et morphismes stratifies", Bull. Amer. Math. Soc. 75 (1969) 240-284. MR 39:970

[11] Sullivan, D., "Singularities in spaces", Proceedings of Liverpool Singularities Symposium (1970), Vol. II, Springer Lecture Notes 209, 1971, pp. 196-206. MR 49:4002

[12] Hironaka, H., "Resolutions of the singularities of an algebraic variety in characteristic zero", Ph.D. Thesis, Harvard University (1963).

[13] Wells, Robert, "Cobordism groups of immersions", Topology 5, pp. 281-294 (1966). MR 33:4946

[14] Milnor, J. (and Kervaire, M.), "Groups of homotopy spheres. I", Ann. of Math. 77 (1963) pp. 504-537. MR 26:5584

[15] Cheeger, Jeff, "On the Hodge Theory of Riemannian pseudomanifolds", Amer. Math. Soc., Proc. Sym. Pure Math. XXXVI (1980) 91-146; and with Goresky and MacPherson, " $L^{2}$ cohomology and intersection homology of singular algebraic varieties", Seminar on Differential Geometry, Yau S.T. (ed.), Princeton Univ. Press, Princeton, NJ, 1982. MR 83a:58081

[16] Morgan, J., and Sullivan, D., "Transversality characteristic class and linking cycles in surgery theory", Annals of Math. 99 (1974) 463-544. MR 50:3240

[17] Goresky, M., and MacPherson, R., "Intersection homology theory", Topology 19 (1980) pp. 135-162; and "Intersection homology II", Inventiones Math. 72 (or 71) pp. 77-130. MR 82b:57010

[18] Novikov, S.P., "Additivity of the signature of manifolds with boundary" (author's web page); and K. Janich, "Characterisierung der signature von Mannifaltijkeiten durch eine Additivitats Eigenschaft", Inventiones Math. 6 (1968) 35-40. MR 37:6954

[19] Singer, I.M., "Prospects in Math", Princeton series (1970).

[20] Sullivan, D., "The signature, geometric cycles, and K-theory", unpublished manuscript, 197374 ; and see [30].

[21] Morava, J., "Extraordinary cohomology theories and K-theory" (author's web page).

[22] Hopkins, M., "Algebraic Topology and Modular Forms", Proceedings of International Congress of Mathematicians, Vol. 1 (Beijing 2002), pp. 291-317, Higher Ed. Press, Beijing, 2002.

[23] Sullivan, D., "Geometric Periodicity and the Invariants of Manifolds" Manifolds, Amsterdam, 1970, Lecture Notes, Springer-Verlag, 197 (1970) 44-75. Also, "Galois Symmetry in Manifolds Theory at the Primes", Proc. ICM, Nice, Part 2 (1970) 169-175. MR 44:2236

[24] Adams, J. Frank, "Stable homotopy theory", lectures delivered at University of California at Berkeley, 1961, Lecture Notes, Springer-Verlag, vol. 3 (notes by Al Vasquez), 1964. MR 32:3061

[25] Hirzebruch, F., "New Topological Methods in Algebraic Geometry", Springer-Verlag, 1956. MR 96c:57002

[26] Weyl, H., Analisis situs combinatorio. Revista Mat. Hispano-Americana, vols. 5-6 (1923-24); reproduced in Ges. Abh., vol. II, pp. 58-59.

[27] Weil, A., "Collected Papers", Vol. II, pp. 555-556; or Amer. J. Math. 79 (1957) pp. 951-952. Correspondence by XXX. MR 80k:01067b 
[28] Yagita, N., "Algebraic structure of cobordism operations with singularities" and "On the Steenrod algebra of Morava K-theory", J. London Math. Soc. (1977), vol. 16, pp. 131-141; and J. London Math. Soc. (1980), vol. 22, pp. 423-438. MR 56:3832

[29] Botvinnik, B., "Manifolds with singularities and the Adams-Novikov spectral sequence", London Math. Soc. Lecture Notes Series, 170, Cambridge University Press, 1992. MR 93h:55002

[30] Siegel, P., "Witt spaces: a geometric cycle theory for KO-homology at odd primes", Amer. J. Math., vol. 105, no. 5, pp. 1067-1105, October 1983. MR 85f:57011

[31] Buoncristiano, S., Rourke, C.P., and Sanderson, B.J., "A Geometric Approach to Homology Theory", London Math. Soc. Lecture Note Series, 18, Cambridge University Press, 1976. MR 54:1234

[32] Chern, S.S., Hirzebruch, F., and Serre, J-P. "On the index of a fibered manifold", Proc. Amer. Math. Soc. 8 (1957), 587-596. MR 19:441c

City College (CUNY), 365 Fifth Avenue, New York, New York 10016

E-mail address: dsullivan@gc.cuny.edu 